\title{
Phlegmasia Cerulea Dolens Following Heparin-Induced Thrombocytopenia
}

\author{
Deepesh G. Aggarwal, Shilpa S. Bhojraj', Ali Asgar Behrainwalla², Charu K. Jani, Simin S. Mehta \\ Departments of Critical Care Medicine, ${ }^{1}$ Anaesthesiology and ${ }^{2}$ Cardiovascular and Thoracic Surgery, Saifee Hospital, Mumbai, Maharashtra, India
}

\section{Abstract}

The authors present a case of a 49-year-old woman who underwent coronary artery bypass grafting after suffering from an acute coronary syndrome and later developed phlegmasia cerulea dolens with heparin-induced thrombocytopenia (HIT) and HIT thrombosis (HITT).

Keywords: Coronary artery bypass grafting, cyanosis, heparin, heparin-induced thrombocytopenia and thrombosis, massive proximal venous thrombosis, phlegmasia cerulea dolens, pulselessness

\section{INTRODUCTION}

Heparin-induced thrombocytopenia (HIT) is a life-threatening immune-mediated reaction due to exposure to heparin (unfractionated, low-molecular-weight heparin; ratio 10:1). ${ }^{[1]}$ HIT occurs in approximately 1 in 5000 hospitalized patients. ${ }^{[2]}$ Drug-induced thrombocytopenia due to heparin differs from that seen with other drugs in two ways - the thrombocytopenia is not usually severe, with nadir counts rarely $<20,000 / \mathrm{L}$ and is associated with increased risk of arterial and venous thrombosis. ${ }^{[1]}$

Phlegmasia cerulea dolens (PCD) is a severe form of deep venous thrombosis characterized by severe venous outflow obstruction, marked limb swelling, pain, bluish discoloration, and venous gangrene if the condition is untreated. Etiological factors include malignancy, femoral vein catheterization, HIT, antiphospholipid antibody Syndrome, surgery, heart failure, and pregnancy. PCD is a life and limb-threatening condition with an overall mortality rate of $20 \%-40 \%{ }^{[3]}$

\section{Case Report}

A 49-year-old woman presented with acute coronary syndrome for which she received aspirin, clopidogrel, atorvastatin, enoxaparin, and tenecteplase at another facility. She was then transferred to our institute for further management. She underwent coronary angiography which was suggestive of triple vessel disease. As the patient was

\begin{tabular}{|l|l|}
\hline \multicolumn{3}{c|}{ Access this article online } \\
\hline Quick Response Code: & Website: \\
& www.ijccm.org \\
\hline
\end{tabular}

in acute congestive heart failure (Killip IV) with pulmonary edema, she was intubated and mechanically ventilated. She underwent coronary artery bypass grafting surgery the following day where she received $20,000 \mathrm{U}$ of unfractionated heparin.

On postoperative day (POD) 6, the patient developed right pedal edema with cyanosis. Bilateral lower limbs Arterial Doppler was normal. Venous Doppler of the right lower limb was suggestive of thrombosis of the right Common Femoral, Superficial Femoral, Popliteal, and Great Saphenous Veins. She also had progressively worsening thrombocytopenia in which the platelet count dropped from $297 \times 10^{9} / \mathrm{L}$ on day 1 of postenoxaparin exposure to a nadir of $20 \times 10^{9} / \mathrm{L}$ on day 8 . With a suspicion of HIT and thrombosis (HITT), enoxaparin was replaced with fondaparinux on POD 7. She tested positive for HIT type 2 antibodies on day 10 . Her platelets started improving by POD 11 [Figure 1].

On POD 13, two-dimensional echocardiography done was suggestive of a left ventricular thrombus (with an ejection fraction of $25 \%$ ) which resolved completely by POD 44 with anticoagulation. Fondaparinux was bridged with oral Vitamin K Antagonist-Acenocoumarol for HITT and PCD. Once there

Address for correspondence: Dr. Simin S. Mehta, Intensive Care Unit, Saifee Hospital, Mumbai, Maharashtra, India. E-mail:simin_mehta@hotmail.com

This is an open access article distributed under the terms of the Creative Commons Attribution-NonCommercial-ShareAlike 3.0 License, which allows others to remix, tweak, and build upon the work non-commercially, as long as the author is credited and the new creations are licensed under the identical terms.

For reprints contact: reprints@medknow.com

How to cite this article: Aggarwal DG, Bhojraj SS, Behrainwalla AA, Jani CK, Mehta SS. Phlegmasia cerulea dolens following heparin-induced thrombocytopenia. Indian J Crit Care Med 2018;22:51-2. 


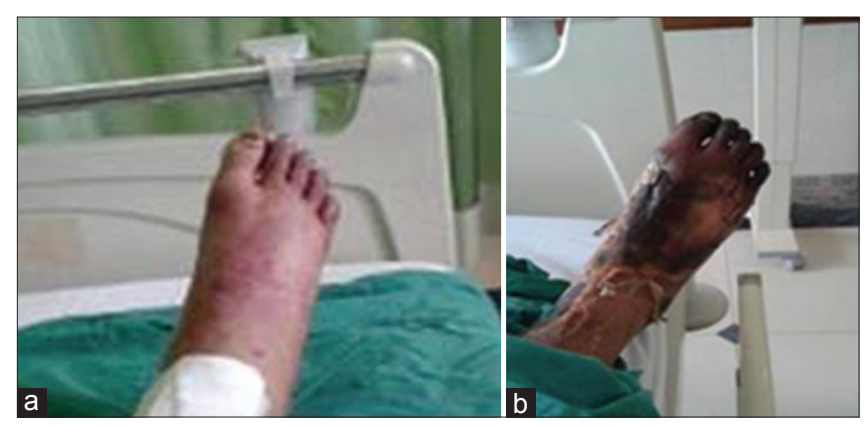

Figure 1: (a) Right Foot edema with peripheral Cyanosis, (b) Right Forefoot Gangrene

was a line of demarcation for gangrene, she underwent a right forefoot amputation on POD 34.

The patient was discharged on POD 47 with medications for cardiac failure along with acenocoumarol.

\section{Discussion}

HIT is an intensely thrombotic, life-threatening immune-mediated reaction caused by platelet-activating antibodies directed against a complex of platelet factor 4 and heparin. ${ }^{[4,5]}$ HIT is characterized by a reduction in the platelet count approximately 4-14 days after the initiation of heparin therapy along with a paradoxical prothrombotic state. The "4Ts" have been proposed as a clinical pretest scoring system for early identification of HIT: thrombocytopenia, timing of symptoms, thrombosis or other sequelae, and no other discernible cause for thrombocytopenia. ${ }^{[2,6,7]}$

Our patient had a combination of clinical presentation, pattern of platelet counts, pretest probability, absence of any drug causing thrombocytopenia, documented heparin-associated antibodies, and a high 4T score of 6 .

PCD is an uncommon form of massive venous thrombosis of extremities due to complete obstruction of both superficial and deep venous return. The resultant severe venous congestion leads to increased arteriolar pressure and tissue ischemia. Capillary involvement is seen in $40 \%-60 \%$ of the cases leading to higher hydrostatic pressure than oncotic pressure resulting in irreversible venous gangrene affecting the skin, subcutaneous tissue, and/or muscle.

The treatment for HITT includes immediate discontinuation of heparin products, start alternative anticoagulation, (and continue for at least 3 months), and not transfuse platelets despite thrombocytopenia. In our case, immediately, after the clinical suspicion of HITT, enoxaparin was replaced with fondaparinux and later bridged with acenocoumarol.

If such a patient requires cardiac surgery in the future, they should be managed with direct thrombin inhibitors. This information should be conveyed to the patient, family members, and emphasized in the medical record, through the inclusion of "heparin allergy."

\section{Financial support and sponsorship}

Nil.

\section{Conflicts of interest}

There are no conflicts of interest.

\section{ReFERENCES}

1. Kasper, DL. Harrisons Principles of Internal Medicine. $19^{\text {th }}$ ed. New York: McGraw Hill Education Medical; 2015. p. 727-8.

2. Greinacher A. Clinical practice. Heparin-induced thrombocytopenia. N Engl J Med 2015;373:252-61.

3. Veltchev LM, Kalniev MA, Todorov TA. Phlegmasia cerulea dolence-risk factors and prevention. Case report. J IMAB Annu Proc 2009;Book 1:89-91.

4. Warkentin TE, Greinacher A. Heparin-induced thrombocytopenia: Recognition, treatment, and prevention: The Seventh ACCP Conference on Antithrombotic and Thrombolytic Therapy. Chest 2004; 126:311S-337S.

5. Rauova L, Zhai L, Kowalska MA, Arepally GM, Cines DB, Poncz M, et al. Role of platelet surface PF4 antigenic complexes in heparin-induced thrombocytopenia pathogenesis: Diagnostic and therapeutic implications. Blood 2006;107:2346-53.

6. Lo GK, Warkentin TE. Preliminary evaluation of a clinical scoring system for estimating the pretest probability of heparin-induced thrombocytopenia: The "4 T's". Blood 2003;102:536a.

7. Ortel TL. Heparin-induced thrombocytopenia: When a low platelet count is a mandate for anticoagulation. Hematology (Am Soc Hematol Educ Program) 2009;1:225-32. 